\title{
Norois
}

Environnement, aménagement, société

\section{Introduction - Afrique(s) atlantique(s). Entre espace de transition et cohérence régionale}

Introduction - Atlantic Africa(s). Between Transition Area and Regional

Coherence

\section{Aziz Ballouche, Evelyne Gauché et Thierry Guineberteau}

\section{OpenEdition}

\section{Journals}

Édition électronique

URL : https://journals.openedition.org/norois/8819

DOI : $10.4000 /$ norois. 8819

ISBN : 978-2-7535-8001-5

ISSN : $1760-8546$

Éditeur

Presses universitaires de Rennes

\section{Édition imprimée}

Date de publication : 20 décembre 2019

Pagination : 7-18

ISBN : 978-2-7535-7904-0

ISSN : 0029-182X

Référence électronique

Aziz Ballouche, Evelyne Gauché et Thierry Guineberteau, «Introduction - Afrique(s) atlantique(s). Entre espace de transition et cohérence régionale », Norois [En ligne], 252 | 2019, mis en ligne le 20

décembre 2019, consulté le 04 janvier 2023. URL : http://journals.openedition.org/norois/8819 ; DOI : https://doi.org/10.4000/norois.8819 


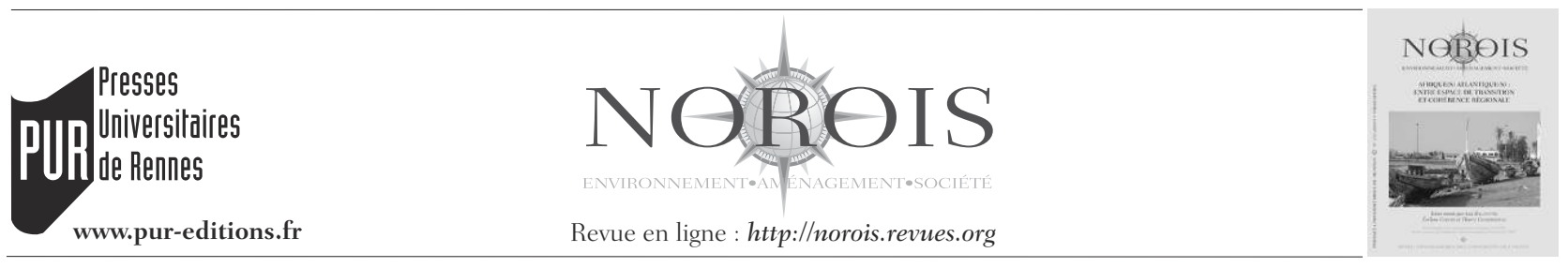

\title{
Introduction \\ Afrique(s) atlantique(s) \\ Entre espace de transition et cohérence régionale
}

\author{
Atlantic Africa(s). Between Transition Area and Regional Coherence
}

\author{
Aziz Ballouche ${ }^{a}$, Évelyne Gauchéb et Thierry Guineberteau ${ }^{c}$
}

a Auteur correspondant, Université d'Angers, UMR 6554 CNRS LETG (aziz.ballouche@univ-angers.fr)
bUniversité François Rabelais, Tours, UMR 7324 CNRS CITERES (evelyne.gauche@univ-tours.fr)
cUniversité de Nantes, UMR 6554 CNRS LETG (thierry.guineberteau@univ-nantes.fr)

Résumé : La façade atlantique du continent africain, entre le Détroit de Gibraltar et le Cap Vert, et ses arrière-pays sont un véritable espace atelier pour comprendre les dynamiques en cours dans cette partie du Monde. Riche de ressources, notamment de la mer, et ouvert sur le monde par une réelle armature portuaire, le Nord-Ouest africain illustre parfaitement les différents aspects des dynamiques géographiques contemporaines : forte littoralisation des activités et des implantations humaines, tension sur les ressources, articulation des enjeux environnementaux et de développement, importance des mobilités et des migrations, compétitions et convoitises dans la mondialisation. Espace de transition par ses milieux et son peuplement, c'est aussi un territoire à la recherche d'une cohérence régionale.

Abstract: The Atlantic coast of the African continent, between the Strait of Gibraltar and the Cap Vert, and its hinterlands are a relevant workshop area to understand the dynamics underway within this part of the world. North West Africa is well-endowed with resources, including maritime, and is open to the world thanks to many ports. This area perfectly illustrates the different aspects of contemporary geographic dynamics: strong population pressure on the coast (activities and human settlements), pressure on resources, a necessary articulation of environmental and development issues, the importance of human mobility and migrations, competitions in a context of globalisation. It is a transition area by its environments and its people, but also a territory in search of a regional coherence.

Mots clés : Afrique - façade atlantique - climatologie - ressource - peuplement - réseau - maritimité - territorialité régionale

Keywords: Africa - atlantic coast - climatology - resource - settlement - network - maritimity - regional territoriality 


\section{INTRODUCTION}

En 2017, les concours nationaux de l'agrégation de géographie et du CAPES d'histoire-géographie ont mis au programme la question suivante : «L'Afrique : du Sahel et du Sahara à la Méditerranée ». Une telle question n'a pas manqué de susciter de nombreuses interrogations sur la pertinence d'une telle délimitation et la cohérence de ce territoire (Andrieu, 2017; Choplin et al., 2017; Fournet-Guérin et Magrin, 2018). Comme l'ont souligné Chopin et al. (2017), considérer l'ensemble des espaces que constituent le désert du Sahara, sa bordure sahélienne et les territoires méditerranéens comme cohérents, ne va pas de soi. Cela ne se défend ni d'un point de vue environnemental, puisque l'on passe d'un espace méditerranéen de moyennes latitudes tempérées au monde intertropical, ni humain, où peuvent être convoquées les traditionnelles divisions entre Afrique blanche, de culture arabo-berbère méditerranéenne, et Afrique noire, héritière de traditions ancrées dans une histoire précoloniale, souvent peu ou mal connue. Cette séparation est aujourd'hui reformulée par l'expression Afrique subsaharienne. En proposant un dossier thématique sur la façade atlantique de cet espace, il fallait tout autant assumer ce grand écart.

Si ces auteurs peuvent regretter que le cadre d'analyse n'ait pas embrassé un espace plus large (du golfe de Guinée à la Méditerranée par exemple), ils insistent souvent sur une logique où, autour du Sahara, les tensions multiformes entre circulations et cloisonnements, intégration et fragmentation, offrent une clé de lecture pertinente pour comprendre les dynamiques territoriales qui animent spécifiquement le Nord-Ouest africain. Il nous semble qu'une telle logique est singulièrement opérationnelle sur sa façade atlantique.

Si les bornes nord et sud d'un espace qui irait du Détroit de Gibraltar au Cap Vert sont évidentes, se limiter à un linéaire côtier n'aurait pas de sens; ce qui justifie d'y intégrer l'arrière-pays. Effectivement, au-delà du littoral, on peut affirmer qu'une forme de maritimité marque les territoires par une forte polarisation des activités et des flux, dont les effets dépassent parfois les pays concernés. C'est ce que nous proposons ici d'argumenter en adoptant un regard sous des angles multiples : via l'environnement, les ressources, les réseaux et les circulations ou encore les tentatives et perspectives d'intégration régionale ou plus globale.

\section{UN ESPACE DE TRANSITION}

Les naturalistes ont longtemps considéré le Sahara comme un obstacle, plus ou moins étanche, entre des mondes disjoints. Outre la barrière de l'aridité, il sépare nettement les régions méditerranéennes à pluies d'hiver, dites tempérées, de la zone sahélienne soumise aux pluies d'été de la mousson africaine. Le " grand désert » peut aussi être vu comme une séparation biogéographique entre les faunes et flores holarctiques au nord et celles à affinités tropicales au sud. Dans une logique déterministe, ces discontinuités naturelles pouvaient être traduites en autant de barrières infranchissables ou au franchissement toujours difficile pour le peuplement, l'exploitation, les échanges ou la circulation des hommes. Certes l'hyperaridité bioclimatique du Sahara, l'absence d'écoulement en surface et la rareté de la végétation sont des réalités, mais le "désert » n'est pas une évidence partout et n'a pas existé de tout temps; ce qui se vérifie particulièrement sur sa façade atlantique.

\section{Un effet de façade}

En matière de milieux, c'est d'abord l'océan qui fait la singularité de notre zone d'étude. Aux basses latitudes moyennes et aux latitudes tropicales, entre $32^{\circ} \mathrm{N}$ et $15^{\circ} \mathrm{N}$, qui concernent la façade de l'Afrique entre Détroit de Gibraltar et Cap Vert, l'Atlantique Est est marqué par les caractéristiques classiques à ces latitudes : un flux régulier de l'alizé de NordEst, les courants côtiers qu'il entraîne (courant des Canaries) et par conséquent des remontées d'eaux profondes, froides et riches en nutriments (upwelling). L'effet de l'alizé est pratiquement permanent sur le littoral saharien mais, selon les saisons, avec le balancement latitudinal de l'anticyclone des Açores, il peut se manifester de façon plus ou moins marquée du nord du Maroc, en été, à la Grande côte sénégalaise, en hiver.

Sur le littoral saharien, entre les régions de Goulimine au Maroc et Nouakchott en Mauritanie, ces conditions atténuent à la fois les températures et leur amplitude annuelle. La température moyenne 
annuelle est ainsi de $20,4^{\circ} \mathrm{C}$ à Tan-Tan ou Dakhla et de $21,7^{\circ} \mathrm{C}$ à Nouadhibou ${ }^{1}$. L'amplitude thermique annuelle à Nouadhibou est de $6,4^{\circ} \mathrm{C}$, alors qu'à Atar situé à la même latitude, environ 400 à l'intérieur du continent, elle est de $13,9^{\circ} \mathrm{C}$ pour une moyenne annuelle de $27,7^{\circ} \mathrm{C}$. Bien que moins marqué cet effet d'atténuation agit également au nord et au sud du Sahara, en région méditerranéenne (entre Rabat et Fès, par exemple) ou sahélienne (entre Dakar et Touba). En termes de bioclimat, comme d'ambiance ou de cadre de vie, cet effet est un trait majeur de la façade atlantique de l'Afrique.

En ce qui concerne les précipitations, les situations sont plus contrastées. En zone sahélienne, les pluies essentiellement liées au front de la mousson africaine subissent peu l'effet de façade, alors qu'au Maroc, celui-ci est bien net, surtout conjugué à la barrière montagneuse de l'Atlas et du Rif, qui distingue nettement la partie occidentale assez bien arrosée, des régions orientales trans-atlasiques, plus arides. C'est pourtant sur la côte saharienne que cet effet se lit le plus nettement. Les eaux fraîches de la surface de l'océan aux latitudes tropicales créent une inversion thermique qui entretient la modération des températures à basse altitude mais s'oppose, en même temps, à l'ascendance des masses d'air et inhibe ainsi la formation des précipitations. Pour reprendre les mêmes exemples, la moyenne des précipitations annuelles est de $18 \mathrm{~mm} / \mathrm{an}$ à Nouadhibou, alors qu'à l'intérieur des terres, elle est de $71 \mathrm{~mm} / \mathrm{an}$ à Atar. Paradoxalement, le même phénomène provoque sur la côte saharienne une forte humidité relative qui atténue significativement la sécheresse atmosphérique du désert, et a aussi pu justifier l'expression de « désert brumeux ». Entre l'automne et le printemps, l'humidité relative dépasse souvent $95 \%$ et se manifeste par une importante rosée nocturne et matinale, qui peut parfois compenser l'indigence des pluies, sous forme de précipitations occultes.

Ainsi, si les remontées d'eaux profondes froides et riches en nutriments provoquent une extraordinaire productivité des milieux côtiers et littoraux, elles limitent aussi les précipitations pluvieuses et renforcent ainsi les contraintes de l'aridité continentale. Pourtant les conditions du désert brumeux de la côte saharienne permettent à une végétation

1. [climate-data.org] buissonnante fournie de se développer sur plusieurs kilomètres, voire quelques dizaines de kilomètres, à l'intérieur des terres. Cette «broussaille-steppe » adaptée à ce paradoxe fait du Sahara atlantique une entité biogéographique originale caractérisée aussi par un endémisme floristique qui souligne sa singularité tout en en faisant un espace de transition entre les flores méditerranéennes et tropicales (Lebrun, 2001). Entrent notamment sur le littoral saharien un certain nombre d'espèces méditerranéennes qui descendent vers le sud jusqu'aux latitudes tropicales, comme les Tamarix, et des espèces tropicales qui remontent vers le nord, jusque dans la région de Tan-Tan et Goulimine (Euphorbia balsamifera dans la bande côtière ou Acacia tortilis dans l'arrière-pays). Les mangroves les plus septentrionales du littoral atlantique africain atteignent le Banc d'Arguin en Mauritanie, où elles arrivent au contact des Spartina maritima des marais côtiers tempérés.

\section{Continuités/discontinuités dans le temps long}

L'expression Sahara vert mise en exergue dans de nombreux articles de vulgarisation, si elle relève plus du mythe que la réalité, amène pourtant à s'interroger sur la permanence de la barrière que peut constituer le désert entre l'Afrique méditerranéenne et le Sahel (Ballouche, 2017). On sait qu'après sa mise en place au Sahara à la fin du Pliocène, le désert a connu d'importantes fluctuations au cours des deux derniers millions d'années. Cette histoire est déterminée par les changements climatiques globaux, dont les éléments en jeu sont les rôles respectifs des hautes pressions tropicales et de la convergence intertropicale qui entrainent des variations latitudinales des limites du Sahara.

Ainsi, les époques globalement froides correspondent à des périodes de forte aridité avec une extension spatiale du désert. Au cours de ces périodes, les systèmes dunaires sahariens ont pu s'étendre jusqu'à environ $15^{\circ}$ de latitude, soit bien au sud du Cap Vert, comme il y a 20000 à 15000 ans lors de la période de l'Ogolien. Ces paléo-dunes, aujourd'hui fixées par la végétation des savanes sahéliennes, marquent encore visiblement de leur héritage les paysages du sud-ouest de la Mauritanie et de l'ouest du Sénégal. Ce sont d'ailleurs ces formations qui peuvent aujourd'hui être remises en 
mouvement par la destruction de leur couverture végétale dans les processus de désertification.

Ces périodes alternent avec des phases chaudes, plus humides, marquées par la remontée en latitude des pluies de mousson et l'atténuation du courant des Canaries, au cours desquelles une végétation de type sahélien ou semi-désertique remplace le désert. Pendant ces périodes humides, les végétations de type méditerranéen qui se développaient au Sahara septentrional ne descendaient pas beaucoup plus au-delà, le Sahara occidental étant alors essentiellement sous influence tropicale (Ballouche, 2017). Sur la partie occidentale du Sahara, cette dernière situation semble avoir régulièrement provoqué l'effacement du désert absolu, favorisant non seulement des échanges nord-sud de faune et de flore mais aussi une diffusion du peuplement humain. C'est ce qu'illustre bien la période humide africaine de l'Holocène (AHP), il y a 9000 à 7000 ans, où le désert s'est très fortement rétracté, avec de nombreux lacs et surfaces humides (Watrin et al., 2009; Lézine et al., 2011). À partir de 5500 ans environ, ces conditions favorables reculent en plusieurs étapes et l'aridité se réinstalle pour arriver progressivement à la situation actuelle (de Menocal et al., 2000; Maley et Vernet, 2015).

On voit donc qu'à l'échelle du temps long, le Sahara n'a pas toujours été une barrière infranchissable car ses conditions d'aridité ont varié. Il a même pu être le foyer d'émergence de civilisations qui ont ensuite essaimé vers le Sahel ou la Méditerranée, notamment dans sa partie occidentale au Néolithique (Mannig et Timpson, 2014; Ozainne et al., 2014).

\section{DES RESSOURCES MISES EN EXPLOITATION}

Contrairement à certains schémas déterministes évoqués plus haut, la façade atlantique de l'Afrique entre le Maroc et le Sénégal offre plutôt bien des atouts largement exploités par les sociétés. La grande productivité des eaux fraiches de l'upwelling et les conditions bioclimatiques favorables du littoral et de l'arrière-littoral, mais aussi les possibilités d'ouverture et d'exportation qu'offre une façade maritime sont autant de facteurs de richesse et d'opportunités de mise en valeur.

\section{Les pêches maritimes, fondamentales et fragiles}

Les eaux poissonneuses de cette façade atlantique, liées notamment au phénomène d'upwelling mentionné précédemment, ont depuis longtemps favorisé le développement d'activités halieutiques aujourd'hui bien ancrées dans le paysage, l'économie et les sociétés. Qu'elles soient pratiquées d'une façon sédentaire ou itinérante, côtière ou hauturière, artisanale ou industrielle, les pêches maritimes constituent une ressource majeure de certains secteurs côtiers et non négligeable pour ces tats africains. L'article de Deme, Ricard et Brehmer (dans ce volume) souligne bien au travers de l'exemple sénégalais tout à la fois les évolutions, la diversité et les enjeux actuels. Parallèlement aux statistiques de captures ou à la part des pêches dans le budget national, le rôle majeur de cette activité peut aussi se lire au travers de la structuration de la profession, l'organisation administrative, ou encore les instituts de recherche. Ainsi, et pour ne prendre que cet exemple, l'INRH marocain (Institut national de recherche halieutique) ou l'IMROP mauritanien (Institut mauritanien de recherche océanographique et des pêches) constituent des structures indéniables de connaissance et de développement de la filière.

Ces pêches maritimes se caractérisent ainsi par des communautés et pratiques traditionnelles localisées. Les pêcheurs Imraguen du Banc d'Arguin en Mauritanie en constituent probablement l'un des exemples les plus emblématiques et les mieux connus, notamment du fait de la présence du PNBA (Parc National du Banc d'Arguin). Ces pêches africaines, ce sont aussi les flottilles piroguières nombreuses et modernisées que l'on va retrouver sur de gros «spots » portuaires côtiers tels Nouakchott, Saint-Louis ou au sud de la Petite Côte sénégalaise (Mbour, Joal) et leurs réseaux de distribution et de transformation. Ces pêches atlantiques ont enfin une dimension industrielle pour partie portée par les flottilles des États riverains mais aussi très largement du fait des flottilles étrangères à la région (UE, Chine, etc.). Les enjeux industriels de l'activité sont bien perceptibles dans certains projets de développement de la filière, telle la stratégie marocaine Halieutis, tout comme dans les tensions récurrentes lors des négociations des accords de pêche entre 
États. Le tout conduit à une pression forte sur la ressource et des constats avérés de surpêche, ainsi qu'à une concurrence dans l'accès aux zones de pêche, y compris dans des configurations transfrontalières (Sénégal/Mauritanie par exemple).

Outre les problématiques internes à l'activité halieutique, celle-ci doit faire face à diverses dynamiques marines ou côtières ayant une incidence constatée ou potentielle sur la ressource comme sur les espaces utilisés (Trouillet et al., 2011). Il en est ainsi de la concentration des populations sur les côtes et de l'amplification associée de l'urbanisation. L'espace se fait alors rare et les besoins liés à la filière ne sont pas nécessairement prioritaires. Cet espace peut se faire parfois encore plus « rare » du fait de l'érosion et de la fragilisation de certains secteurs côtiers. En la matière, le quartier des pêcheurs de Saint-Louis au Sénégal est tout à fait caractéristique des dynamiques à l'œuvre et à venir. Cette concentration littorale du bâti dans un contexte de difficile gestion des réseaux s'accompagne de risques aigus de pollution des eaux marines, risques accentués lors des fortes précipitations qui lessivent et évacuent vers la mer les déchets solides. Par ailleurs, les volontés réaffirmées de développement du transport maritime (cf. "Stratégie Portuaire Nationale 2030 » du Maroc, cf. réorganisation des terminaux portuaires à l'échelle du Grand Dakar) ont une incidence tout autant sur la partie terrestre (artificialisation, besoin d'espaces portuaires, réseaux) que sur la partie maritime (accès, zones d'attente, risques de pollution). Enfin, les perspectives en matière d'exploitation off-shore d'hydrocarbures, au large de la Mauritanie et du Sénégal, ne sont pas sans soulever des interrogations sur l'avenir de l'activité halieutique.

La préservation de la ressource est donc un élément central de la pérennisation de l'activité halieutique. La mise en place d'AMP (aires marines protégées) ou de ZPP (zones de pêche protégées) telles que présentée pour le cas sénégalais dans l'article de Deme, Ricard et Brehmer s'inscrit pour partie dans cette logique. Mais plus largement, par son ancrage, son poids et sa fragilité liée à l'extraction d'une ressource vivante, les pêches maritimes sont au cœur d'enjeux de gestion qui les dépassent nécessairement et sur lesquels nous reviendrons en dernière partie.

\section{Tourisme : le soleil et la mer...}

Tout aussi emblématique de la façade atlantique, le tourisme balnéaire est une voie sur laquelle ont misé ces pays pour développer leur économie, particulièrement le Maroc et le Sénégal, plus que la Mauritanie. Constitué d'alternances de plages sableuses, de secteurs à falaise et de zones humides riches en biodiversité ce littoral dispose de nombreuses potentialités pour attirer des touristes et fonder une économie touristique balnéaire. C'est la voie qu'a suivie le Sénégal où le tourisme balnéaire, encouragé par l'État au début des années 1960 dès l'Indépendance, est devenu un véritable moteur de l'économie, au second rang après la pêche (Diombéra, 2012). Suivant la vogue de la promotion de nouvelles destinations balnéaires «tropicales » pour répondre aux enjeux du tourisme de masse des années 1970 et dans un contexte de sécheresse ayant affecté la production arachidière et les rentrées de devises (ibidem), le littoral sénégalais est passé d' "un état répulsif à un état attractif » par le biais d'un développement touristique centré sur quatre pôles : Dakar, Saint-Louis, Thiès et la Basse Casamance (Diombéra, 2010).

Si le Maroc a également fait du tourisme un secteur prioritaire de ses choix économiques dès la fin des années 1960, il a d'abord été considéré à l'époque comme une destination pionnière sur la rive sud de la Méditerranée (Troin, 2002). Depuis, ce tourisme balnéaire a plutôt stagné sur la côte méditerranéenne et il s'est développé et maintenu dans le Sud atlantique (Agadir, Essaouira), laissant s'imposer dans le pays un tropisme touristique balnéaire malgré une composante culturelle marquée. En témoigne le lancement en 2001 du Plan Azur visant à attirer 10 millions de touristes aux alentours de 2013, grâce à la réalisation de six stations balnéaires, dont une sur le littoral méditerranéen (Saïdia) et cinq sur le littoral atlantique (du nord au sud : Port Lixus, Mazagan, Mogador, Taghazout et Plage blanche, respectivement à proximité des villes de Larache, El Jadida, Essaouira, Agadir et Tan Tan).

Entre ces deux pays au développement touristique particulièrement avancé sur les littoraux, la Mauritanie, au contraire, n'est pas une destination balnéaire. Bien que le tourisme soit toujours resté en retrait dans l'économie nationale, le pays avait misé 
sur le Sahara, et particulièrement l'Adrar (d'Atar à Ouatane en passant par Chinguetti, anciens relais sur la route des caravanes) autour des activités de trekking, randonnées chamelières, circuits en $4 \times 4$ (Choplin et Roullier, 2006). Depuis 2007 et le premier attentat contre des touristes français à Aleg puis la montée en puissance du terrorisme djihadiste au Sahara, ce secteur est en fort recul.

Au-delà du strict crédo du tourisme balnéaire - la mer et le soleil -, le patrimoine naturel et culturel, notamment en lien avec la maritimité, est aussi un atout important pour ce secteur dans notre zone d'étude. C'est le cas de plusieurs villes plus ou moins touristiques de la côte atlantique marocaine : Tanger, Asilah, El-Jadida, Safi ou Essaouira. Si Asilah affiche son slogan « ne pas bronzer idiot » autour d'un festival culturel depuis les années 1980, El-Jadida, par exemple, station balnéaire à fréquentation principalement nationale, a adopté après l'inscription de sa cité portugaise au Patrimoine mondial de l'Unesco en 2004, une stratégie de promotion internationale basée, entre autres, sur cet argument. Dans le même esprit, le patrimoine historique de Saint-Louis du Sénégal, également inscrit sur la liste de l'Unesco, est le support d'une activité touristique couplée à l'attractivité naturelle et paysagère du fleuve.

Le tourisme de nature et plus largement l'écotourisme constituent une activité encore limitée mais qui peut avoir un réel potentiel. Si au Maroc elle demeure généralement articulée à des formes de tourisme sportif, notamment sports de glisse ou de pêche, en Mauritanie et surtout au Sénégal, elle peut constituer un secteur à part entière. L'exemple principal en est le Parc National des Oiseaux du Djoudj, dans le Delta du fleuve Sénégal, inscrit sur la liste des zones humides d'importance internationale (Convention de Ramsar) et site du Patrimoine mondial de l'Unesco depuis 1981 (Taïbi et al., dans ce volume). Avant la dégradation de la situation sécuritaire en Mauritanie dans les années 2010, le Parc National du Banc d'Arguin, également inscrit sur les listes de la Convention de Ramsar et du Patrimoine mondial, pouvait aussi illustrer ce type de tourisme, mobilisant moins de fréquentation mais offrant un « produit » à très grande valeur patrimoniale naturelle. Cependant, les retombées économiques sur les populations locales sont loin d'être satisfaisantes, tout comme les conflits avec les objectifs de préservation des écosystèmes et de la faune qui sont à l'origine de la création des aires protégées.

Ainsi, ces pays d'Afrique atlantique misent tous, d'une façon ou d'une autre et à des degrés divers, sur le développement touristique. Cependant, cette stratégie a ses limites, tant géopolitiques que socioéconomiques ou encore environnementales. Le développement touristique y a fortement pâti des événements terroristes des dernières années. La Mauritanie, par exemple, est loin aujourd'hui d'avoir recouvré le niveau de fréquentation d'avant la crise sécuritaire (Châtelot, 2018). La diversification et un manque de renouvellement des activités sont aussi une faiblesse du tourisme balnéaire, comme au Sénégal où il avait été noté une régression depuis le début des années 2000 (Diombéra, 2012). Au Maroc, les liens avec l'arrière-pays, souvent montagneux et abritant un riche patrimoine culturel, tendent à se développer et un certain tourisme de masse est désormais en train d'y perdre du terrain face au produit culturel (El Affas, 2019). Par ailleurs, ce tourisme balnéaire de masse est, dans l'ensemble de la région, à l'origine d'une fragilisation des littoraux et des écosystèmes. De ce fait, les stations littorales, comme celles du plan Azur marocain, peinent à se développer, notamment à cause de leur difficulté à attirer les investisseurs, manifestant le besoin pour l'industrie touristique de se renouveler et pour ces pays de mettre en place de nouvelles stratégies touristiques plus durables.

\section{Des richesses agricoles et minières}

Si les productions agricoles et de matières premières ne sont pas géographiquement déterminées par la façade atlantique du Nord-Ouest africain, leur contribution à l'économie des trois pays étudiés ici est, en revanche, à mesurer à l'aune de leur capacité à exporter. Ainsi, aux côtés des agricultures vivrières paysannes et aux systèmes agro-pastoraux traditionnels, chacun de ces pays porte une image habituellement associée aux stéréotypes que sont les productions d'exportation qui les distinguent: agrumes et primeurs pour le Maroc, arachide pour le Sénégal et, dans une moindre mesure élevage bovins pour la Mauritanie. Les agricultures de ces territoires sont cependant en pleine mutation et les politiques agricoles se renouvellent. 
Depuis 2008, dans le cadre de la modernisation de l'agriculture marocaine, le Plan Maroc Vert (PMV) a entamé un processus de spécialisations des régions marocaines en bassins de production, dans le cadre de plans agricoles régionaux (PAR). Un des objectifs en est la «modernisation » de 4 Offices régionaux de mise en valeur agricole pilotes (ORMVA), dont trois sur la façade atlantique (Doukkala, Loukos et Gharb), centrant notamment l'ouest-atlantique sur la production des grandes cultures industrielles (céréales, canne à sucre...) à la fois pour couvrir le marché national et pour l'export. La région d'Agadir, le Souss, est quant à elle déjà anciennement tournée vers la production d'agrumes, en partie pour l'exportation. Le Sahara n'est pas en reste de ce processus de modernisation de l'agriculture, comme dans la région de Dakhla où la culture sous serres était de 450 ha fin 2009, avec une production hors sol à fort rendement à l'hectare de plus de 31000 tonnes de primeurs, projetée à 1000 ha à l'horizon 2020 (Quarante, 2014). Dans une analyse récente des 10 ans du PMV, un analyste de l'IRIS, soulignait un certain nombre de succès permettant aux entreprises de l'agro-industrie marocaine d'être plus compétitives et d'exporter vers l'Europe, mais aussi en Afrique (Abis, 2019). Il considère également que le PMV peut constituer une source d'inspiration de politique agricole pour des pays du continent africain, comme le Sénégal. D’ailleurs, le volet agricole Plan Sénégal Emergent (PSE) lancé par le président Macky Sall en 2014 est clairement calqué sur le PMV en capitalisant sur l'expérience marocaine. Le PSE affiche une volonté de mettre en valeur le potentiel de l'agriculture commerciale, au premier chef l'arachide tout en affirmant vouloir continuer à développer l'agriculture familiale. D'autres analyses plus critiques reprochent cependant à ces plans leur propension productiviste et techniciste, ignorant les dérives ultra-libérales et les effets environnementaux, notamment sur l'eau, et conteste les impacts réels sur l'emploi et la croissance (Akesbi, 2012).

Sur un autre registre économique, dans le Rif occidental marocain, la culture du cannabis est en train de connaître son développement le plus profond depuis que sa culture de masse a commencé au début des années 1980. L'adoption de variétés à haut rendement et le développement de pratiques agricoles modernes se traduisent par la production de dérivés du cannabis plus variés et plus puissants, et des quantités croissantes de haschich envoyées en Europe (Chouvy et Macfarlane, 2018).

Contrairement au golfe de Guinée ou au large de l'Angola, riches en gisements d'hydrocarbures offshore, la façade atlantique du Nord-Ouest africain a longtemps fait office de parent pauvre. Ce n'est que très récemment, que les prospections au large de la Mauritanie et du Sénégal ont révélé d’importantes réserves gazières sur le talus continental entre 1000 et $3000 \mathrm{~m}$ de profondeur. Malgré une succession d'espoirs et de désillusions au cours des dernières années, un accord sénégalo-mauritanien a été conclu en 2018 pour permettre l'exploitation commune du gisement Grand Tortue Ahmeyim (GTA) à l'échéance 2021, concédée à l'entreprise pétrolière britannique British Petroleum. En attendant l'entrée réelle des deux pays dans le giron des producteurs de gaz et de pétrole, l'arrière-pays de la façade atlantique nord-ouest africaine possède surtout d'importantes richesses minières (phosphates, fer, cuivre et plus récemment hydrocarbures...) faisant l'objet d'une exploitation poussée et bénéficiant de débouchés portuaires pour l'exportation. Les phosphates au Maroc ou le fer et le cuivre en Mauritanie, sont les moteurs historiques du développement des grandes villes portuaires de Casablanca ou de Nouadhibou (Port Étienne). Sous le protectorat français, le port de Casablanca a d'abord été conçu par le Maréchal Lyautey pour exporter les phosphates de la région de Khouribga. Depuis, le Maroc (en incluant le Sahara occidental) est devenu le deuxième producteur et premier exportateur mondial de phosphates et détient $70 \%$ des réserves mondiales ${ }^{2}$. Ce secteur est animé par le premier groupe industriel du pays, l'Office Chérifien des Phosphates (OCP) qui affichait, en 2018, un chiffre d'affaires 4,1 milliards d'euros. De même, en Mauritanie les industries extractives constituent le principal secteur exportateur, avec $27 \%$ du PIB en 2017, dont $15 \%$ pour le minerai de fer de Zoueirat.

Les richesses minières et pétrolières peuvent aussi être la pierre d'achoppement entre les États, comme au Sahara occidental, où elles exacerbent la discorde entre le Maroc et l'Algérie. Dans des pays où elles constituent la base d'une économie rentière, comme pour la Mauritanie, elles peuvent aussi être source d'une vulnérabilité aux exportations.

2. Source USGS, 2018, [www.usgs.gov/centers/nmic/phosphate-rock-statistics-and-information]. 


\section{UNE LITTORALISATION GÉNÉRALISÉE ET À TOUTES LES ÉCHELLES}

Peuplement, villes, centres de décisions et activités se concentrent de plus en plus en zone littorale, ici comme dans d'autres régions du monde. Tant au Maroc, qu'en Mauritanie et au Sénégal, les capitales se situent sur le littoral atlantique, qui concentre aussi l'essentiel des activités industrielles. Au Maroc, plus de $60 \%$ des industries du pays et $80 \%$ des emplois industriels nationaux se situent sur l'axe Kenitra-Safi (Nakhli, 2010), avec un centre de gravité autour de sa capitale économique Casablanca. A l'échelle de ce vaste pays peu peuplé qu'est la Mauritanie, l'évolution de la ville de Nouakchott est tout à fait caractéristique de cette tendance lourde et accélérée : sa population est en effet passée de 2000 habitants en 1960 à près d'un million aujourd'hui (soit 1/4 de la population du pays) pour une ville-capitale créée ex nihilo lors de l'Indépendance.

Plus largement, le rôle d'interface que joue aujourd'hui la façade atlantique est d'autant plus remarquable qu'à travers l'histoire moderne, jusqu'à la colonisation européenne, cette zone était restée à l'écart des grands courants commerciaux, malgré l'existence de villes côtières historiques. L'essentiel des échanges transsahariens s'appuyaient, depuis le Moyen Âge, sur les grandes cités intérieures, Fès et Marrakech au nord, Tombouctou et Djenné au sud, avec des étapes sahariennes comme Oualata ou, plus tard, Tindouf. Sur les côtes, les comptoirs portuaires coloniaux (Larache, Mazagan, Saint-Louis) pouvaient certes être des portes d'ouverture sur le monde mais aussi des points de crispation souvent assiégés et coupés de leurs arrière-pays.

\section{Ports et réseaux comme marqueurs de la littoralisation}

En Afrique du Nord-Ouest, comme nous l'avons vu plus haut, ce sont historiquement les principaux ports exportateurs de matières premières qui sont les plus importants. Aujourd'hui, sur cette côte atlantique, la concurrence est rude entre les principaux ports, qui ne lésinent pas sur les moyens pour devenir des plates-formes de référence sur le continent (Miossec, dans ce volume).
La logique historique d'exportation de matières premières se maintient à Nouadhibou et caractérise encore des ports comme celui de Laâyoune, au Sahara occidental sous administration marocaine, pour les phosphates de Boukraa, ou celui de Nouakchott pour le cuivre d'Akjoujt. Le port phosphatier de Jorf Lasfar est, en revanche, associé à un important complexe industrialo-portuaire transformant l'essentiel de la production en engrais et en acides, et exporte donc principalement des produits finis ou semi-finis.

Cependant, la grande mutation du transport maritime qui vise à intégrer pleinement cette façade atlantique dans la mondialisation est étayée par la conteneurisation (Miossec, dans ce volume). L'exemple emblématique en est le port TangerTanger Med, sur le détroit de Gibraltar, qui a relégué celui de Casablanca au deuxième rang des ports marocains. Construit de 2004 à 2007, ce grand complexe portuaire, nouveau hub maritime marocain, a été conçu pour être le plus grand port de transbordement africain, en articulation étroite avec le grand terminal espagnol d'Algésiras. Avec 3,3 millions de conteneurs EVP traités en 2017, il est devenu le $45^{\mathrm{e}}$ port du monde et le premier port d'Afrique. Le trafic des porte-conteneurs y est complété par un trafic roulier de camions semi-remorques et de remorques. Dans une moindre mesure, le port de Dakar bénéficie aussi d'une position géographique exceptionnelle à l'intersection des lignes maritimes reliant l'Europe à l'Amérique du Sud, l'Amérique du Nord à l'Afrique du Sud. Il est aussi la porte d'entrée d'un trafic important vers un arrière-pays qui s'étend jusqu'au Mali.

Les grands ports s'intègrent dans une stratégie de développement et d'intégration régionale dont témoignent les grandes infrastructures de communication récentes ou en projet. Afin de mieux assurer la complémentarité avec Tanger-Med, une liaison ferroviaire à grande vitesse est entrée en service, en 2018, entre les villes de Casablanca et de Tanger. La LGV Tanger-Casablanca ramènera le trajet entre ces deux villes à $2 \mathrm{~h} 10$ au lieu de $4 \mathrm{~h} 45$ actuellement, et la même liaison est également envisagée pour assurer le fret rapide nocturne. Il est également en projet, à l'horizon 2025, le prolongement de cette ligne « atlantique » vers Marrakech, puis jusqu’à Agadir sur 900 km. Ce TGVM (TGV Maghrébin) s'inscrit en relation avec un projet, peut-être uto- 
pique ressortant régulièrement des tiroirs, de tunnel ferroviaire sous-marin entre l'Espagne et le Maroc.

De Tanger également partent désormais deux axes routiers majeurs. La « rocade méditerranéenne ", qui relie cette ville portuaire à l'ensemble du littoral méditerranéen jusqu’à la frontière algérienne, désenclave tout le littoral de la montagne rifaine à travers pour l'essentiel des régions rurales marginales, l'un des principaux objectifs étant le développement touristique (Gauché, 2010). Vers le sud, l'axe autoroutier qui relie Tanger à Agadir, via Marrakech, se poursuit ensuite par une route de qualité qui suit le littoral saharien, jusqu'à Nouadhibou, Nouakchott et traverse le fleuve Sénégal au bac de Rosso et secondairement au barrage de Diama.

\section{Les enjeux de la gestion territoriale}

Cette littoralisation constatée et à venir trouve ses limites notamment sur le plan environnemental. Ainsi au Maroc, l'embouchure de l'oued Sebou draine les rejets domestiques, industriels et agricoles de l'arrière-pays (régions de Fès, Meknès, Sidi Kacem, Sidi Slimane, Kenitra); l'axe industriel Mohammedia-Casablanca-El Jadida-Safi est particulièrement touché par la pollution en raison de la forte concentration urbaine et industrielle, de même que la baie d'Agadir où s'ajoutent les effets du tourisme de masse (Nakhli, 2010). À Nouakchott, situé en partie en dessous du niveau de la mer, le risque d'inondation et de submersion représente une menace venue de l'Atlantique. Plusieurs inondations (incursions marines, remontées de nappes) au cours des 20 dernières années ont causé des dommages importants aux infrastructures et bâtiments, et la rupture du cordon dunaire pourrait conduire à la submersion d'une grande partie de la ville (Sow, 2019). Le littoral de Nouakchott connaît en effet une dynamique érosive importante consécutive à l'aménagement de la jetée du port en 1986. Le trait de côte a progressé de plus de $800 \mathrm{~m}$ en amontdérive alors qu'au sud de l'épi, en aval-dérive, la côte a reculé de plus de 500 m (Faye et al., 2008). Malgré les mesures prises pour consolider le littoral, il faut toutefois constater que la situation de Nouakchott s'est aggravée au cours de ces dernières années en raison des effets du changement climatique, mais aussi à cause de l'activité humaine et de la difficulté à maîtriser l'urbanisation rapide et informelle.
Un autre exemple de ces enjeux environnementaux, celui des périmètres territoriaux des aires protégées, est développé dans ce volume (Taïbi et al.) à propos de la réserve de biosphère transfrontière du Bas Delta du Sénégal (RBTDS), entre Mauritanie et Sénégal.

Sans être exclusive d'autres considérations, la question environnementale devient de fait l'un des moteurs de l'évolution des conceptions et tentatives de gestion des territoires. Comme nous l'avons déjà ponctuellement abordé dans ce texte (AMP, patrimoine, etc.), cette évolution s'opère dans un mixe d'initiatives endogènes et de fortes incitations internationales. Mais les enjeux de gestion sur cette façade maritime sont plus vastes et peuvent être déclinés au travers quelques points majeurs.

Il s'agit d'abord de dépasser l'approche sectorielle ou tubulaire pour aller vers des approches territoriales : face aux plans/stratégies pêche, tourisme, transport maritime, il est nécessaire de parvenir à concrétiser des règles et principes généraux d'aménagement des zones côtières. C'est ce que tente d'expérimenter la loi relative au littoral de 2015 au Maroc ou le Plan d'aménagement du littoral mauritanien (PDALM). Il faut par ailleurs parvenir à dépasser la "rupture » terre/mer afin d'avoir une véritable intégration de la partie maritime dans les réflexions d'aménagement, une continuité maritimeterrestre de l'entité géographique à comprendre et gérer. Parallèlement, l'intégration de l'hinterland est indispensable afin d'éviter une accentuation de la déconnexion entre des littoraux dynamiques et des arrière-pays en stagnation, voire en déclin/abandon. Il faut enfin concevoir des processus rénovés et efficaces d'aménagement permettant l'intégration de nouveaux acteurs dans les réflexions, décisions, et mises en application (pouvoirs locaux, société civile, etc.) tout en ayant une capacité à agir et réagir vite dans des configurations où les basculements peuvent s'opérer très rapidement (cf. Laouina, Taïbi et al. et Deme et al. dans ce volume).

Dans les dynamiques territoriales de cette façade maritime, un dernier enjeu majeur est celui de l'articulation et de la mise en cohérence d'échelles différentes : approches locales, approches nationales et celles supranationales, qu'elles soient à l'échelle de la sous-région, de la façade africaine, du Maghreb ou de l'Afrique de l'Ouest ou qu'elle relève d'un processus plus global de mondialisation. 


\section{Réorganisation régionale et mondialisation}

En bornant notre espace d'étude entre le Détroit de Gibraltar et le Cap Vert nous y avons intégré l'arrière-pays qui permet de comprendre son organisation et son fonctionnement. Il faut aussi envisager un avant-pays pour saisir à la fois une part des héritages historiques de l'organisation territoriale mais surtout les profondes mutations que ces territoires connaissent à l'heure de la mondialisation. Au-delà de son rôle d'interface, la façade atlantique est vectrice d'intégration régionale comme nous l'avons déjà abordé concernant les axes routiers. Objectivement, les logiques de l'intégration régionale sont pourtant contrastées.

L'héritage historique et culturel, notamment par rôle civilisationnel unificateur de traditions islamiques communes, est un atout non négligeable et se maintient jusqu’à nos jours par des flux non négligeables d'étudiants, aussi bien vers les universités modernes que vers les centres traditionnels de formation religieuse au Maroc et en Mauritanie. Ces réseaux sont aussi réactivés par les chemins qu'empruntent les flux de migrants subsahariens vers l'Europe, mais dont une partie aboutit aujourd'hui vers une destination finale au Maroc, volontairement ou involontairement (Laouina dans ce volume).

Des obstacles majeurs à cette réorganisation régionale demeurent cependant à travers les tensions entre États, et particulièrement entre les deux puissances régionales que sont le Maroc et l'Algérie. La question de Sahara occidental contribue au cloisonnement politique et géopolitique de la sous-région. Si l'ex-colonie espagnole est intégrée depuis 1976 au Maroc, tant sur le plan administratif qu'en termes d'aménagement ou d'exploitation des ressources (cf. phosphates de Boukraa ou équipement touristique de Dakhla), la souveraineté marocaine est contestée juridiquement. Les conséquences sur les relations du Maroc avec l'Algérie sont désastreuses, mais elles sont aussi causes de tensions régulières avec la Mauritanie ou d'autres pays africains. Concrètement, l'érection d'un mur de défense qui double la frontière internationale avec la Mauritanie crée un obstacle hermétique entre les deux pays qui limite les passages aux points frontières entre Lagwira et Nouadhibou au Cap Blanc. Paradoxalement, c'est aussi cette question qui a justifié une diplomatie marocaine offensive sur le continent africain.

La dynamique de renforcement des relations diplomatiques et commerciales du Maroc avec de nombreux pays africains, et en premier lieu le Sénégal, vise à la fois à se constituer un réseau d'alliance dans les discussions internationales pour l'achèvement du processus de décolonisation du Sahara ex-espagnol, menées par l'ONU, mais a aussi des raisons économiques réelles. Si les échanges commerciaux du Maroc avec l'Afrique subsaharienne restent faibles au regard de ceux qu'il entretient avec l'Europe, ils connaissent une très forte croissance et un solde excédentaire, accompagné d'un réel effort d'investissement (Laouina dans ce volume). Dans ce processus, la demande d'adhésion du Maroc à la Communauté Économique des États de l'Afrique de l'Ouest (CEDEAO) est un pas important.

Au côté de ces dynamiques chaotiques, à l'échelle sous-régionale, la façade atlantique de cette partie de l'Afrique est également concernée par différentes facettes de la mondialisation. Nous l'avons signalé à propos du rôle que jouent les ports dans les mutations du transport maritime (Miossec dans ce volume) ou encore par le biais du tourisme international. La région est aussi un important nœud dans différents réseaux de trafics plus ou moins licites (migrants, cannabis). Ceux-ci mettent toujours en exergue la place majeure que tient aujourd'hui le Détroit de Gibraltar dans un système-monde globalisé. De fait, qu'il s'agisse de flux de biens ou de personnes, le Maroc est ainsi devenu une charnière de l'articulation Europe/Afrique intimement liée aux deux continents. En témoigne, par exemple, la réserve de biosphère intercontinentale de la Méditerranée (RBIM), englobant un vaste domaine terrestre sur les deux rives du détroit, entre Andalousie en Espagne et Péninsule tingitane au Maroc, mêlant contrôle et protection (Goeury, 2009). D'autres tentatives de réduction de l'effet de frontière naissent sur ce littoral aux richesses menacées, comme la réserve transfrontière du BasDelta du Sénégal, déjà évoquée (Taïbi et al., dans ce volume).

Enfin, les enjeux de la mondialisation concernent aussi aujourd'hui des flux immatériels, d'informations, comme l'illustre très bien l'article de Mihoubi (dans ce volume) sur le soft-power de Radio Chine Internationale (RCI). Avec des relais 
FM en Mauritanie et au Sénégal, RCI développe ses propres systèmes de médias comme dans toute l'Afrique, afin de proposer la vision chinoise de l'actualité internationale. Dans des pays où la radio reste parfois le médium le plus utilisé, ce rôle n'est pas négligeable pour venir concurrencer l'influence des anciennes puissances coloniales et promouvoir sa propre puissance économique. Cet exemple est révélateur de l'émergence de nouveaux acteurs politiques et économiques sur la scène africaine, longtemps chasse gardée dans la lignée des héritages coloniaux.

\section{Conclusion}

Les littoraux sont le siège de dynamiques puissantes qui traduisent diversement l'expansion de l'économie mondiale et les processus d'intégration régionale Nord-Sud ou Sud-Sud (Semmoud, 2009). Cette section du littoral atlantique qu'est le Nord-Ouest africain n'échappe pas à ces logiques et concentre de nombreux facteurs de dynamique, propres à une littoralisation marquée (à l'instar d'autres parties du globe), comme à une intégration encore en devenir des littoraux et de leurs arrièrepays, porteuses d'enjeux multiples.

Ces dynamiques lui confèrent une certaine cohérence régionale que l'on peut lire au travers de problématiques communes (prise en compte des questions environnementales, gestion des activités existantes, maîtrise des activités émergentes, prospectives démographique et urbaine, etc.). Les ressources marines de cette façade atlantique, du Maroc au Sénégal, partout exploitées par les sociétés, confèrent aussi à cet espace une relative continuité. On a également l'émergence de liens transnationaux (réseaux routiers, mobilité étudiante, organismes transfrontaliers à l'image de l'Organisation pour la mise en valeur du fleuve Sénégal (OMVS) ou les réserves transfrontalières de biosphère, accords économiques tels l'accord sénégalo-mauritanien sur mise en exploitation des hydrocarbures off-shore, etc.). Mais cette " cohérence » est en chantier et l'équilibre demeure fragile. Les territoires concernés sont aussi en concurrence économique (attractivité portuaire, accès aux ressources halieutiques, captation des flux touristiques, etc.), voire en tensions géopolitiques - le Sahara occidental reste sur ce point une épine -, et ce dans un contexte où l'affir- mation de son indépendance, comme la création de ressources et d'emplois demeurent pour chacun des objectifs prioritaires.

D'autres logiques territoriales peuvent conduire à qualifier cet ensemble d'espace de transition. Cela renvoie bien évidemment à la traditionnelle, voire désuète, différentiation Maghreb et Afrique Noire, ou au passage du monde méditerranéen au monde tropical, mais la transition peut aussi concerner le continuum de la terre à la mer, laissant apparaître la nécessité d'appréhender dans l'interaction des entités géographiques continentales et océaniques. Comme peuvent en témoigner les migrations, les transitions sont à la fois internes et externes à cet espace.

Cohérence géographique ou espace de transition, la compréhension et la gestion des dynamiques à l'œuvre passent nécessairement par la capacité à mener de front des réflexions multiscalaires, à pouvoir articuler les logiques supranationales (qu'elles soient à l'échelle de la sous-région ou de l'ensemble de cette façade atlantique), nationales (par définition très variables suivant la puissance et la stabilité des États concernés), et locales, cette dernière dénomination renvoyant à une très grande variété de taille et caractéristiques spatiales, d'une agglomération urbaine côtière comme Dakar jusqu’à une vaste aire protégée comme le Banc d'Arguin.

\section{Bibliographie}

Abis S., 2019. Maroc : l'agriculture comme levier de développement national et international. [www.iris-france. org/130463-maroc-lagriculture-comme-levier-de-developpement-national-et-international/].

Akesbi N., 2012. Une nouvelle stratégie pour l'agriculture marocaine : Le «Plan Maroc Vert ». New Medit, vol. 11, $\mathrm{n}^{\circ} 2$, p. 12-23.

Andrieu J. (dir.), 2017. L'Afrique: du Sahel et du Sahara à la Méditerranée, Ellipses, 312 p.

Ballouche A., 2017. Le Sahara : barrière ou trait d'union entre Méditerranée et Sahel? in Andrieu J. (dir.), L'Afrique. Du Sahel et du Sahara à la Méditerranée, Ellipses, p. 41-54.

Châtelot C., 2018. Dans le désert mauritanien, le tourisme renaît tout doucement, Le Monde, 9/12/2018, [https://www.lemonde.fr/afrique/article/2018/12/09/ dans-le-desert-mauritanien-le-tourisme-renait-tout-doucement_5394903_3212.html].

Choplin A., Marei N., Pliez O., 2017. L'Afrique du Sahel et du Sahara à la Méditerranée, éd. Atlande, 236 p. 
Choplin A., Roullier L., 2006. Tourisme et politique en Mauritanie ou comment (re)visiter le Sahara : l'exemple de l'Adrar mauritanien, Les Cahiers d'Outre-Mer, 233, p. 29-50.

Chouvy P.A., Macfarlane J., 2018. Agricultural innovations in Morocco's cannabis industry, International Journal of Drug Policy, 58, p. 85-91.

De Menocal P., Ortiz J., Guilderson T., Adkins J., Sarnthein M., Baker L., Yarusinsky M., 2000. Abrupt onset and termination of the African Humid Period: rapid climate responses to gradual insolation forcing, Quaternary science reviews, 19, p. 347-361.

Diомве́ra M., 2010. Aménagement et gestion touristique durable du littoral sénégalais de la Petite Côte et de la Basse Casamance, Thèse de doctorat en tourisme, Université Gaston Berger, Saint-Louis (Sénégal), 343 p.

Diombéra M., 2012. Le tourisme sénégalais à la recherche d'une nouvelle identité, Téoros, vol. 31, n² 2, p. 21-30.

El Affas A., 2019. Tourisme balnéaire : Mais que deviennent les stations du plan Azur?, L'Économiste, 5537, 18/06/2019. [https://www.leconomiste.com/article/1046508-tourismebalneaire-mais-que-deviennent-les-stations-du-plan-azur].

Faye I.B. Nd., Hénaff A., Gourmelon F., Diaw A.T., 2008. Évolution du trait de côte à Nouakchott (Mauritanie) de 1954 à 2005 par photo-interprétation, Norois, 208, p. 11-27.

Fournet-Guérin C., Magrin G., 2018. L'Afrique, du Sahel et du Sahara à la Méditerranée : intégrations, circulations et fragmentations, Bulletin de l'association des géographes français, vol. 95, n² 2, p. 165-174.

Gauché E., 2010. Le désenclavement des territoires ruraux marginalisés du Nord du Maroc : les conséquences de la nouvelle route côtière méditerranéenne dans le Rif oriental (territoire des Beni Saïd), Norois, 214, p. 67-84.

Goeury D., 2009. Protéger ou contrôler le détroit? La réserve de la biosphère intercontinentale de la Méditerranée Andalousie (Espagne)-Maroc in Semmoud B. (dir.), Mers, détroits et littoraux : charnières ou frontières des territoires?, Paris, L'Harmattan, p. 271-285.

Lebrun J.P., 2001. Introduction à la flore d'Afrique, éd. Quae, $221 \mathrm{p}$.
Lézine A.-M., Hély C., Grenier C., Braconnot P., KrinNer G., 2011. Sahara and Sahel vulnerability to climate changes, lessons from Holocene hydrological data, Quaternary Science Reviews, vol. 30, nº 21-22, p. 3 001-3 012.

Maley J., Vernet R., 2015. Populations and climatic evolution in North Tropical Africa from the end of the Neolithic to the dawn of the Modern Era, African Archaeological Review, vol. 32, $\mathrm{n}^{\circ}$ 2, p. 179-232.

Manning K., Timpson A., 2014. The demographic response to Holocene climate change in the Sahara, Quaternary Science Reviews, 101, p. 28-35.

Nakhli S., 2010. Pressions environnementales et nouvelles stratégies de gestion sur le littoral marocain, Méditerranée. Revue géographique des pays méditerranéens, 115, p. 31-42.

Ozainne S., Lespez L., Garnier A., Ballouche A., Neumann K., Pays O., Huysecom E., 2014. A question of timing: spatio-temporal structure and mechanisms of early agriculture expansion in West Africa, Journal of Archaeological Science, 50, p. 359-368.

Quarante O., 2014. Si riche Sahara occidental : poissons, primeurs, phosphates exportés vers l'Europe, Le Monde diplomatique, 720 , p. 7.

Semmoud B. (dir.), 2009. Mers, détroits et littoraux : charnières ou frontières des territoires?, Paris, L'Harmattan, 413 p.

Sow B., 2019. Nouakchott, colmatage des cordons dunaires, Traversées mauritanides, [http://traversees-mauritanides. com/articles/afficher/226].

Troin J.F. (dir.), 2002. Maroc. Régions, pays, territoires, Paris, Maisonneuve et Larose, 502 p.

Trouillet B., Guineberteau T., Bernardon M., Le Roux S., 2011 . Key challenges for maritime governance in West Africa: Fishery-based lessons from Guinea and Mauritania, Marine Policy, vol. 35, n 2, p. 155-162.

Watrin J., Lézine A.M., Hély C., Contributors, 2009. Plant migration and plant communities at the time of the "green Sahara”, Comptes Rendus Geoscience, 341, p. 656-670. 